\title{
Source and pattern identification of ground deformation based on the framework of Blind Source Separation-Nonnegative Matrix Factorization: a case study in a long-term GPS monitoring mine
}

hongyu Gu ( $\nabla$ xy0909040129@126.com )

chengdu center, china geology survey https://orcid.org/0000-0001-6837-2685

fengshan Ma

Chinese Academy of Sciences

Liangjun Lin

China Geological Survey

donghui wang

China Geological Survey

yongbo Tie

China Geological Survey

weichang Chen

Chinese Academy of Cultural Heritage

\section{Research Article}

Keywords: source and pattern identification, Nonnegative Matrix Factorization, ground deformation, GPS

Posted Date: March 22nd, 2021

DOl: https://doi.org/10.21203/rs.3.rs-232646/v1

License: (c) (i) This work is licensed under a Creative Commons Attribution 4.0 International License.

Read Full License 
Source and pattern identification of ground deformation based on the framework of Blind monitoring mine

4

Hongyu $\mathrm{Gu}^{* 1}$, Fengshan $\mathrm{Ma}^{2}$, Liangjun $\mathrm{Lin}^{* 4,5}$, Donghui Wang ${ }^{1}$, Yongbo $\mathrm{Tie}^{1}$, Weichang $\mathrm{Chen}^{3}$

1. Chengdu Center, China Geological Survey, Sichuan, Chengdu 610081

2. Key Laboratory of Shale Gas and Geoengineering, Institute of Geology and Geophysics Chinese Academy of Sciences, Beijing 100029

3. Chinese Academy of Cultural Heritage, Beijing 100029

4. Tianjin Center, China Geological Survey, Tianjin 300170

5. Xiong'an Urban Geological Research Center of China Geological Survey, Beijing 100037

Corresponding author: Hongyu Gu \& Liangjun Lin

E-mail: xy0909040129@126.com; 9781866765@qq.com

Address: Yihuanlu Beisanduan No.2, Chengdu, China, 610081

Fengshan Ma

E-mail:1083471408@.qq.com

Liangjun Lin

E-mail: 9781866765@qq.com

Donghui Wang

E-mail: 742824902@qq.com

Yongbo Tie

E-mail: 414429287@qq.com

Weichang Chen:

E-mail: 289835829@qqq.com

\begin{abstract}
Ground deformation caused by mining affects the safety of underground mining and buildings. High-resolution characterization of ground deformation respond to latent sources is a first step toward improved hazard forecasting. We rely on long-term GPS displacement data and an improved NMF algorithm of BSS to identify the sources driving ground deformation. The NMF identifies three sources (S1, S2, and S3) with distinct spatial and temporal surface deformation patterns and quantitatively reveals the contribution of each source to ground deformation. S1 captures horizontal Y-displacement related to horizontal tectonic stress $\sigma_{1}$. S2 dominates vertical Z-displacement with a widespread isotropous deformation driven by self-weight body force. S3 controls horizontal Xdisplacement related to horizontal tectonic stress $\sigma_{2}$. Besides, we find that independent contributions of these three sources can be resolved from the GPS data. The results show that the sharp change of source contribution and even transformation of dominate source are highly related to the severe deformation belt. This phenomenon is time independent, and helps us to select sites and find the potential risk area at its early stage.
\end{abstract}

Keywords: source and pattern identification; Nonnegative Matrix Factorization; ground deformation; GPS 


\section{Introduction}

Ground deformation is an common phenomenon in terms of civil engineering, groundwater pumping, earthquake, landslide and underground mining operations (Cao et al. 2019; Gourmelen et al. 2007; Schultz-Fellenz et al. 2020; Ye et al. 2016; Zhao et al. 2012) and always leads to severe damage of buildings and even collapse of roof caving (Ding et al. 2017; Xia et al. 2019). Typically, there are multiple sources (factors) that can cause rock movement, such as topography, type of rock, structure of rock mass, tectonic stress, mining technology, and co-seismic vibration effects, etc. The combination of these sources and the dominant source vary significantly depending on the local geological conditions. Without greater confidence in how these proposed factors affect ground deformation near surface, we cannot reliably use the results of geodetic analyses to infer shallow deformation, nor can we formulate models to predict deformation in future events.

In recent years, these sources have been widely studied by various methods to have a deep understanding of the mechanism of ground deformation. There are four broadly categories.

First, numerical model based on finite element or discrete element method. It is a forward method that takes some sources (e.g., stress condition, joint distribution, and mining operations) and their generalized parameters into consideration (Fathi Salmi et al. 2017; Kabwe 2017; Khanal et al. 2015; Salmi et al. 2019). The number and kinds of input sources are always decided by prior knowledge and generalized parameters from laboratory. Although numerical model is parameters dependent and sensitive, researchers can, to some extent, identify the dominant source depend on the fluctuation of displacement and stress by inputting different parameters of one source while fixing other parameters at the same time. The second category is classical statistical methods (e.g., correlation analysis, factor analysis, regression analysis, etc.). These methods try to find the law of ground deformation from observed data(Hui et al. 2018). They also can help to find out parts of the sources leading to ground deformation. However, the hidden sources of massive data with high dimensions cannot be easily interpreted by using these methods (Sahu et al. 2017; Song et al. 2011). The third category is analytical solution based on empirical methods, profile function, and influence function(Díaz-Fernández et al. 2010). These methods take the geometrical and mechanical parameters as input to predict the ground deformation. The type and value of parameters are highly dependent on the prior knowledge of researchers. However, it is unreasonable to predict before we know the mechanism of each potential source causing ground deformation. The forth category is machine learning consists of a series of supervised learning algorithms(Chen et al. 2017; Perrin et al. 2015; Zhou et al. 2019). These methods use a set of data to train the learning model with factors identified by prior knowledge and then to fit the observed data. Therefore, these methods are not self-constraint by data and much dependent on the knowledge of researchers.

However, none of these studies try to objectively and quantitatively find out that 1) what kind of and how many sources leading to the ground deformation? 2) what is the independent pattern of each source? 3) how much is the proportion of each source contributing to the deformation at a specific site? and 4) how the dominant source transfers with time among these sources?

Mechanism and pattern vary significantly among different sources when causing ground deformation. As a result, the characteristics of ground deformation induced by different sources can be also different in terms of horizontal and vertical displacement. In plain words, even though we did not know who they (sources) are, how many of them there and how they did (causing ground deformation), but what they have done was recorded in the data. Based on this logic, it is possible to find a backward way to separate the sources from the long-term monitored displacement data. If 
sources can be successfully decomposed from observed data, hidden pattern of each source can be revealed independently with knowledge of petrology, tectonic geology, fracture mechanics, and rock mechanics, etc. Most importantly, it is the transformation of dominant sources with time in a concerned area that is essential to the prediction of ground deformation and safety management.

Indeed, sources identification causing temporal and spatial development of ground deformation is still challenging. In recent years, source identification has received extensive attention in areas such as signal processing, biomedical engineering, data analysis and data mining. Nonetheless, the theory and applications are still being developed. Source identification can be complex because 1) some of source signals may mutually interfere, 2)there may be attenuation (linearly or nonlinearly) of signals when transmitting through the medium or discontinuity, 3 ) the ratios of signal to noise vary in real word, 4) some of sources may be partly collinear or have similar patterns.

The observed displacement data can be seen as the mixture signal of different displacement patterns with some unknown mixing processes. From this point of view, we can introduce Blind Source Separation (BSS) in the field of signal processing to find the hidden sources and their patterns. The aim of BSS is to process observations acquired by sensor arrays in such a way that the original unknown source signals are extracted by various algorithms without knowing or with limited information about the characteristics of the transmission channels through which the sources propagate to the sensors. Therefore, BSS can be used to reveal hidden sources and patterns in a mixed data set.

The basic framework of BSS can be described as a linear mixture model by equation 1 .

$$
X=A S+E
$$

Where $\boldsymbol{X} \in R^{m \times n}$ is observed data (mixtures). $\boldsymbol{A} \in R^{m \times p}$ is an unknown mixing matrix representing the linear combination of the sources and each row of $\boldsymbol{S} \in R^{p \times n}$ is a source. $\boldsymbol{E}$ represents additive noise or interference introduced during mixing and transmission.

Typically, the only known information in BSS is the mixtures $\boldsymbol{X}$. We need to determine the number of sources $\boldsymbol{S}$ and the solution of mixing matrix $\boldsymbol{A}$ and $\boldsymbol{S}$, i.e., mathematically, we need to solve equation 2

$$
\vartheta=\min \|\boldsymbol{X}-\boldsymbol{A S}\|_{F}^{2}
$$

It is noteworthy that such a problem is ill-posed and has an infinite number of solutions. Thus, extra imposed constraints (e.g., statistical independence, non-negativity, sparsity, maximum mutual information, etc.) and regularized optimization procedures are often required to minimize the objective function $\vartheta$ and to find an optimal and robust solution. Different constraints are usually imposed on $\boldsymbol{A}$ and $\boldsymbol{S}$ based on particular cases and may lead to different results. Many methods are included in BSS (e.g., independent component analysis (ICA), sparse component analysis (SCA), non-negative matrix/tensor factorization (NMF/NTF), etc.). They all can successfully identify the hidden sources and patterns from observed data set. However, it is the physical meaning and interpretation of particular case that curial to determine which method to be used.

ICA is one of the early and widely used techniques for BSS. It is an extension of linear principal component (PCA), where mutually orthogonal assumption is replaced by a stronger statistically independent assumption of latent sources (Ciaramella et al. 2006). Cohen-Waeber et al. (2018) applied ICA to InSAR time series in San Francisco East Bay Hills and revealed four distinct spatial and temporal surface deformation components (sources). However, the performance of ICA is not good in some areas (e.g., in bio-signal and genomic signal) because the latent sources are partially 
or entirely correlated. In other words, it is hard and often impossible to verify the statistical assumptions on the sources(Tangirala et al. 2007). Under this situation, much effort has been devoted to find an alternative approach with weaker assumptions. SCA started to emerge at the end of the 1990s and became prominent during the 2000s(Chang et al. 2006; Lee et al. 1999).Unlike ICA, SCA is suited to correlated sources and tries to explain data as a mixture of sparse components(Karoui et al. 2012). In practice, both non-negativity and sparsity are often desirable or necessary when the hidden sources have physical meaning. However, results of ICA and SCA often lead to the subtraction in order to reconstruction of observed data. As a result, it is difficult to interpret the physical meaning of sources.

In contrast to ICA, NMF enforces a non-negative constraint exhibiting some degree of natural sparsity on the mixing matrix $\boldsymbol{A}$ and source matrix $\boldsymbol{S}$ (Lee and Seung 1999). In other words, NMF does not allow subtraction and combinations, and, therefore, it is often used to quantitatively describe the parts that comprise the entire entity. Furthermore, matrix factorization methods that exploit non-negativity and sparsity constraints usually lead to estimation of the hidden sources with specific structures and physical interpretations, in contrast to other BSS methods. For example, it is the sources that contribute to the development (rather than reduce) of ground deformation that gains our most concerns. Sparsity constraint significantly limits the number of sources to represent the observed ground deformation data. Non-negativity constraint gives the physical interpretation of each source on the deformation at monitoring sites.

In this study, the highlight is that without knowing other subsurface conditions and other prior knowledge, we introduce and improve Blind Source Separation (BSS) from the field of signal processing to identify the latent sources and their corresponding patterns based only on a long-term transient displacement data collected by GPS between 2001 and 2017 (measuring interval: 0.5 year). Nonnegative Matrix Factorization (NMF) algorithm (one of BSS algorithms) by Lee and Seung (1999) is applied to decompose the displacement data. Finally, we obtain the following items: 1) the number of identified sources, source patterns and physical meanings, 2) the dynamic of dominant source by comparing the contribution of each source.

\section{Methods}

All the main steps of the method are shown in Fig. 1. We develop an extended NMF framework to decouple the ground deformation data. This method consists of (1) definition of physical problem in a mathematical way, (2) extended NMF with stronger sparsity constraints, (3) initialization of NMF, (4) determination of true number of sources, and (5) Evaluation and selection of robust solution.

\subsection{Definitions}

Let us consider ground deformation is recorded by $m$ GPS monitoring sites at a fixed measurement frequency. Ground deformation is subjected to the effects of $p$ unknown physical sources that cause rock movement. The locations of the sources are unknown. Physical process of different sources may interfere through the medium and their influences may be distributed or point. In our analysis, there are no assumptions neither on the initial or boundary conditions like in numerical model nor stochastic or probability on physical process. The only assumption in this particular problem is that the value of ground deformation at a motoring site is the proportionally added by unknown sources.

Here, we give the mathematical form of this particular problem. 


$$
\boldsymbol{X}_{m \times n}=\boldsymbol{A}_{m \times p} \boldsymbol{S}_{p \times n}+\boldsymbol{E}_{m \times n}
$$

Where $\boldsymbol{X}_{m \times n}$ is observed data by $m$ GPS sites and $n$ monitoring campaigns. $\boldsymbol{A}_{m \times p}$ is an unknown mixing matrix representing the linear combination of $p$ sources. $\boldsymbol{S}_{p \times n}$ is a source contribution at each discrete time moment. $\boldsymbol{E}_{m \times n}$ represents the measurement errors or noises. The number of hidden sources is definitely less than the number of GPS monitoring sites (i.e., $p \leq m$ ).

\subsection{NMF with sparsity constraints}

In order to estimate matrix $\boldsymbol{A}$ and $\boldsymbol{S}$, a cost function $\vartheta$ is chosen to quantify the similarity measure (also referred to as divergence of dissimilarity, distance depending on the probability distribution of estimated sources and the structure of data) between reconstructed data and observed data. $\|\cdot\|_{F}^{2}$ represents the Frobenius norm which is the squared Euclidean distance. $\alpha_{A}$ and $\alpha_{S}$ are nonnegative regularization parameters (typically, $\alpha_{A}=\alpha_{S} \in[0.01,0.5]$ ) and the sparseness increases with an increase in the values of regularization coefficients. Penalty terms $J_{A}(\boldsymbol{A})$ and $J_{S}(\boldsymbol{S})$ enforce a certain application-dependent features to get a unique and desired solution.

$$
\begin{gathered}
\vartheta=D_{F}(\boldsymbol{X}|| \boldsymbol{A} \boldsymbol{S})=\frac{1}{2}|| \boldsymbol{X}-\boldsymbol{A S} \|_{F}^{2}+\alpha_{A} J_{A}(\boldsymbol{A})+\alpha_{S} J_{S}(\boldsymbol{S}) \\
\text { s.t. } a_{i j} \geq 0, s_{j t} \geq 0, \forall i, j, t
\end{gathered}
$$

In order to impose sparsity, we choose to use $\ell_{1}$-norm to minimize the cost function

$$
\begin{gathered}
\vartheta=D_{F}(\boldsymbol{X}|| \boldsymbol{A} \boldsymbol{S})=\frac{1}{2}|| \boldsymbol{X}-\boldsymbol{A S}\left\|_{F}^{2}+\alpha_{A}\right\| \boldsymbol{A}\|\|_{1}+\alpha_{S}\|\boldsymbol{S}\|_{1} \\
\text { s.t. } a_{i j} \geq 0, s_{j t} \geq 0, \forall i, j, t
\end{gathered}
$$

To minimize the cost function $\vartheta$, we choose the multiplicative update algorithm (equation 6 , 7) developed by Lee and Seung (1999), which updates the $a_{i j}$ while keeping $s_{j t}$ fixed, and then updates the $s_{j t}$ while keeping $a_{i j}$ fixed. Obviously, all the successive estimates remain positive if the initial estimate is positive. However, if an element of matrix becomes zero, it remains at zero for all the successive iterations. To circumvent this problem, we force the values of $a_{i j}$ and $s_{j t}$ not to be less than a small positive value $\varepsilon$ (typically, $\varepsilon=10^{-16}$ ).

$$
\begin{aligned}
a_{i j} & \leftarrow a_{i j} \frac{\left[X S^{T}\right]_{i j}-\alpha_{A}}{\left[A S S^{T}\right]_{i j}+\varepsilon} \\
S_{j t} & \leftarrow s_{j t} \frac{\left[A^{T} X\right]_{j t}-\alpha_{S}}{\left[A S S^{T}\right]_{j t}+\varepsilon}
\end{aligned}
$$

The iterative algorithm is stopped when there is little or no improvement of cost function between successive iterations, $\omega=10^{-10}$.

$$
\Delta \vartheta=\frac{\left|D_{F}^{(k)}-D_{F}^{(k-1)}\right|}{D_{F}^{(k)}} \leq \omega
$$

\subsection{Optimization and Solution}

Obviously, the final solution is almost a local minima because the global minima is seldom achievable. In order to achieve a reasonable and optimal solution, another three issues (the first issue is to develop a problem dependent cost function) in NMF should be stressed: 1) alleviation of the rotation indeterminacy, 2) determination of the number of sources, 3) initialization of $\boldsymbol{A}$ and $\boldsymbol{S}$.

\section{Alleviation of the rotation indeterminacy:}

The eq. 3 can also be presented as $\boldsymbol{X}_{m \times n}=\boldsymbol{A}_{m \times p} \boldsymbol{R} \boldsymbol{R}^{-1} \boldsymbol{S}_{p \times n}+\boldsymbol{E}_{m \times n}$. It is easy to find many rotational matrixes $\boldsymbol{R}$ such that $\boldsymbol{A}_{m \times p} \boldsymbol{R}=\widehat{\boldsymbol{A}}$ and $\boldsymbol{R}^{-\mathbf{1}} \boldsymbol{S}_{p \times n}=\widehat{\boldsymbol{S}}$ are also the solutions of eq. 3 . Therefore, the solution of NMF is non-unique. To overcome this, we should keep the input data $\boldsymbol{X}$, decomposed matrixes $\boldsymbol{A}$ and $\boldsymbol{S}$ sufficiently sparse (Rickard and Cichocki 2008). One common 
way is to normalize the input data $\boldsymbol{X}$ to make $\boldsymbol{A}$ and $\boldsymbol{S}$ zero-grounded. Besides, it is a necessary step to find a meaningful approximation of the hidden pattern for ground deformation neglecting the scaling ambiguity.

\section{Number of sources:}

Remember that the number of sources (rank of $\boldsymbol{S}, p$ ) and mixing process is unknown in NMF. Therefore, the number of sources can be extracted from observed data only. However, this remains an open issue. There are several methods by trial and error and heuristic techniques for determining the number of sources. 1) $p$ is determined by the dimension of data, i.e., $p<m n / n+n$, where $m$ represents the number of observations and $n$ represents the number of monitoring campaigns. 2) $p$ can be chosen in an ideal noiseless case by increasing the number of sources until the error of observed and reconstructed data $\left(\boldsymbol{E}=\boldsymbol{X}-\boldsymbol{X}^{\prime}\right)$ is zero. 3) Tangirala et al. (2007) proposed a PSV method based on the incremental variance captured by each source relative to the total power to determine the value of $p .4$ ) others such as residual analysis can also help to choose the rank(Cuss et al. 2016).

In this work, a heuristic method proposed by Minka (2001) was used to calculate the smoothness index defined as:

$$
S I(k)=\frac{\operatorname{var}\left[\left\{\widetilde{\lambda}_{i}\right]_{i=k+1}^{I-1}\right]}{\left.\operatorname{var}\left[\tilde{\lambda}_{i}\right\}_{i=k}^{I-1}\right]}, k=1,2, \ldots, I-2
$$

where $\tilde{\lambda}_{i}=\lambda_{i}-\lambda_{i+1}, \lambda$ is the eigenvalue of the covariance matrix for observed data in a descending order $\left(\lambda_{1} \geq \lambda_{2} \geq \cdots \geq \lambda_{I}>0\right)$. The sample variance is defined as:

$$
\operatorname{var}\left[\left\{\tilde{\lambda}_{i}\right\}_{i=k}^{I-1}\right]=\frac{1}{I-k} \sum_{i=k}^{I-1}\left(\tilde{\lambda}_{i}-\frac{1}{I-k} \sum_{i=k}^{I-1} \tilde{\lambda}_{i}\right)^{2}
$$

Finally, the number of sources $p$ can be selected by the following criterion:

$$
p=\arg \min _{k=1,2, \ldots, I-2} S I(k)
$$

\section{Initialization:}

The intrinsic of multiplicative update algorithms is nonconvex, even though the cost function is strictly convex with respect to one set of variables (i.e., either $\boldsymbol{A}$ or $\boldsymbol{S}$ ). Therefore, it is impossible to find an analytical-based optimal solution(Rezaei and Boostani 2013). The solution and convergence are highly depending on the initialization of $\boldsymbol{A}$ and $\boldsymbol{S}$. Initializations nearing the optimal point in search space can enhance the performance of NMF, while poor initializations often lead to low convergence, and, in some certain instances, incorrect results. Many initialization methods have been proposed, such as random, PCA, SVD, divergence-based k-means, and IGA(Boutsidis and Gallopoulos 2007; Langville et al. 2014; Xue et al. 2008). To obtain a robust initialization of $\boldsymbol{A}$ and $\boldsymbol{S}$, we use the following steps:

1) Generate $k$ initial $\boldsymbol{A}$ and $\boldsymbol{S}$ with Monte Carlo stimulation and run the ALS algorithm to obtain initialized $\boldsymbol{A}$ and $\boldsymbol{S}$ (Bertin et al. 2007) for predetermined rank $p$ in eq. 11.

2) Run the proposed algorithm in this paper for each set of initial matrices.

\subsection{Evaluation criteria of solutions}

All the possible solutions are evaluated on the basis of criteria in terms of relative error $(e)$, orthogonality $(o)$ and sparsity $(u)$ of identified sources(Hoyer 2004). Each of these criteria reveals the capability of the demixing method from a specific aspect. The final optimal solution is a tradeoff among these three criteria, and this can be defined as a Euclidean distance in a 3D rectangular coordinate system. 


$$
\zeta=\min \left(\left\|\boldsymbol{v}_{i}\right\|^{2}\right), i=1,2, \ldots, p *(k+1)
$$

Where $\boldsymbol{v}_{i}$ is a vector composed of the three parameters $\left(e_{i}, o_{i}, u_{i}\right) . e_{i}, o_{i}$ and $u_{i}$ are defined as the following equation 13 to 15 with a monotonic property. The value of $e_{i}, o_{i}$ and $u_{i}$ ranges $[0,+\infty),[0,+\infty)$, and $[1,+\infty)$, respectively. A lower value of each parameter represents a better performance in terms of a specific aspect.

$$
\begin{gathered}
e_{i}=\|\boldsymbol{X}-\boldsymbol{A} \boldsymbol{S}\|^{2} /\|\boldsymbol{A}\|^{2} \\
o_{i}=\left\|\boldsymbol{O}_{\boldsymbol{i}}\right\|^{2}, \boldsymbol{O}_{\boldsymbol{i}}=\left(O_{1}, O_{2}, \ldots, O_{l}\right), O_{l}=\boldsymbol{S}_{q}{ }^{T} \boldsymbol{S}_{h}, l=C_{p}^{2}, 1 \leq q \neq h \leq p \\
\left\{\begin{array}{c}
u_{i}=\left\|\boldsymbol{U}_{\boldsymbol{i}}\right\|^{2}, \boldsymbol{U}_{\boldsymbol{i}}=\left(U_{1}, U_{2}, \ldots, U_{p}\right) \\
\text { where } U_{p}=\frac{\sqrt{n}-1}{\sqrt{n}-\left(\Sigma\left|s_{p h}\right|\right) / \sqrt{\sum s_{p h}^{2}}}, h=1,2, \ldots, n
\end{array}\right.
\end{gathered}
$$

\subsection{Permutation of identified sources}

Methods based on NMF cannot give the order of identified sources measured by explained variance like PCA. However, one of our goals is to identify the dominant source that is crucial for the prediction and controlling of ground deformation. The dominant source should explain the most ground deformation, and the secondary source explains the second-most remaining ground deformation. In order to reorder the identified sources, we defined the contribution $\xi_{\iota}$ by $\iota$ th source as the following equation 16. The source with the highest value of $\xi_{\iota}$ is the most robust one that can be seen as the dominant source. By using this measurement, sources are reordered in a decreasing order of contribution.

$$
\xi_{\iota}=\frac{\left\|A_{i p} S_{p j}\right\|^{2}}{\|X\| \|^{2}}, i=1,2, \ldots, m ; j=1,2, \ldots, n
$$

\section{Application}

\subsection{Geological setting}

Jinchuan No.2 mine (JinChang City of GanSu Province, China) is one of the largest underground nickel mine in the word (Fig. 2a). Mining area lies in a monoclonal structure and more than 180 oblique faults distribute in this area after experiencing each geological tectonic movement and magma intrusion since Luliang Movement of Protozoic in China (Hui et al. 2018). High-level tectonic stress is a prominent characteristic in the mine area. In shallow strata, the axis of the maximum principal stress is horizontal (NE), which is basically perpendicular to the strike of the ore body (strike : $50^{\circ} \mathrm{W}$, and dip angle : $40^{\circ}$ to $70^{\circ}$ ). The maximum principal stress axis rotates to SSW-SW direction in deep strata. The dip of maximum principal stress also shows difference from shallow to deep strata. The dip increases up to $40^{\circ}$ with the depth increasing (Cai 1999; Zhao et al. 2012). Ultrabasic rocks are the ore-bearing native rock. Surrounding rock comprises of granite, marble, and migmatite. These rock masses have poor mechanical stability because of the high density of discontinuity. For more detail in geological conditions, see Li et al. (2004) and Li et al. (2020a). The mechanized backfill mining technique is adopted in this mine field because of the well-developed geological foliation in the metamorphic strata and poor massif stabilities. After finishing of excavation in an access drift, the void is backfilled by cement paste subsequently and then the next access drift is excavated. Thus, no interval pillar is left and the filling body in the upper layer is served as the artificial roof for mining of the next layer. Underground mining operations have induced large scaled ground deformation 
and surface fissures after 1999. Therefore, GPS has been used to monitor the ground deformation since 2001. Previous studies have qualitatively assessed that high tectonic stress, mining rate, discontinuity in rock mass, ratio of backfill and mining were the factors significantly affecting the ground deformation(Ma et al. 2015; Zhao et al. 2013).

\subsection{Data processing}

GPS monitoring network contains a reference net and a deformation monitoring net on the ground surface. Reference net containing 7 benchmarks is set far away from the deformed area. During the long-term period, some of points are destroyed. Therefore, a total of 78 monitoring points with successive time series out of 101 monitoring points are selected in this study. These points distribute along the exploratory lines with a spacing of $50 \mathrm{~m}$ between two points on the same line. Besides, an additional monitoring line ( $\mathrm{H}$ line) is laid out on the upper surface of the ore body, which is perpendicular to the exploratory lines (Fig. 2b). Original data is received by Z-12-type GPS receiver and antenna (Ashtech, USA) (Fig. 2c). The nominal accuracies of the horizontal and vertical displacements are $3 \mathrm{~mm}+0.5 \mathrm{ppm}$ and $5 \mathrm{~mm}+1 \mathrm{ppm}$, respectively. Square error of processed displacement data is about $\pm 1.96 \mathrm{~mm}$. For a single monitoring point, displacement data comprises of the increment of X, Y, Z (local coordinate system) and each of them has 30 records. Besides, displacement data is offset without changing the structure of data to ensure there is no negative value in the matrix.

\section{Results}

The GPS monitoring data is a mixture of a series of unknown sources with dynamic mixing ratios. Our goal is to separate the spatial and temporal patterns of dominate long-term and commonmode. The NMF does this by minimizing cost function with non-negative and sparsity constraints. To seek a trade-off between interpretability and statistical fidelity, the formulated evaluation criteria helps to select the optimal solution from all possible solutions that has the highest resolution of each source pattern.

After 100 tries of NMF and a series of optimization criteria by using the parameters of Table 1, we obtain the 100 solutions with different accuracies (Fig. 3). The optimal solution is determined by error $(e)$, orthogonality $(o)$ and sparsity $(u)$ according to eq. 12 with the number of iteration steps of 1245. The value of $e, o$, and $u$ is $0.02,11.14$, and 1.06, respectively. Although the NMF cannot give the accurate solution, the 100 tries show a validation in statistics. The reconstructed data with less noises (Fig. 4c) fits well with original data (Fig. 4a).

The final solution consists of three sources (Fig. 4b), namely source 1 (S1), source 2 (S2), and source 3 (S3), which optimally isolate the major spatial and temporal trends underlying the ground deformation time series. For comparison, the amplitude is normalized by the relative maximum. It is worth noting that these three sources are not orthogonal because NMF does not assume the orthogonality of sources. Therefore, the sum of source contribution is not equal to one according to the equation 16. The source contribution to original data (Table 2) shows that $\mathrm{S} 1$ has the highest contribution of 0.53 , and $\mathrm{S} 3$ has the secondary highest contribution of 0.51 , and $\mathrm{S} 2$ has the lowest contribution of 0.28 .

$\mathrm{S} 1$ is a source that has a high impact on $\mathrm{Y}$ displacement and a low impact on $\mathrm{X}$ and $\mathrm{Z}$ displacement. In contrast, $\mathrm{S} 3$ shows a high contribution to $\mathrm{X}$ displacement and a low contribution to $\mathrm{Y}$ and $\mathrm{Z}$ displacement. S2 primarily contributes to the $\mathrm{Z}$ displacement and has a low contribution to $\mathrm{X}$ and $\mathrm{Y}$ displacement (Fig. $4 \mathrm{~b}$ ).

\subsection{Spatial pattern of source}


The mixing matrix A represents contribution of the three sources at different monitoring point. Therefore, each column of the mixing matrix $\mathbf{A}$ can be regarded as the spatial pattern of one source. As the element of $\mathbf{A}$ is fixed, the spatial pattern of each source is stable among different monitoring campaigns. This means that these three spatial patterns are time-independent and not to be affected by mining intensity and mining methods. The value of displacement in different monitoring campaign is controlled by the magnitude of source transient. Fig. 5 shows the contour of each column of matrix A that represent the spatial pattern of S1 (Fig. 5a), S2 (Fig. 5b), and S3 (Fig. 5c), respectively.

$\mathrm{S} 1$ captures the feature of $\mathrm{Y}$ displacement. In general, the value of $\mathrm{Y}$ displacement decreases from northwest to the southeast. There are two displacement centers that is nearly divided by line 14. The contribution of S1 near line 14 decreases sharply because the contour is dense. This indicates there exists a deformation belt that has different mechanical properties near line 14 causing the sharp change of $Y$ displacement.

$\mathrm{S} 2$ controlling the $\mathrm{Z}$ displacement describes a homogeneously and broadly distributed pattern. A spatial shape like concentric circle shows the effect of gravity and it seems that the displacement does not affected by many kinds of discontinuities. This indicates that NMF has successfully extracted a gravity-induced pattern. Another important conclusion is that the highest contribution of S2 locates at the edge and gradually decrease at the center. This suggests that the vertical displacement at the center is not gravity-induced.

S3 controlling the $\mathrm{X}$ displacement exhibits two concentric circle shapes at southeast and northwest direction. The contribution of S3 increases from northwest to the southeast. Like the pattern of S1, the two concentric circles are combined by a strong deformation belt distributing near the $\mathrm{H}$ line and the center of the two circle are on the opposite side.

\subsection{Physical meaning of identified source}

In study area, high tectonic stress has been empirically considered as an important factor to the ground deformation(Ma et al. 2015). However, the mechanism of ground deformation induced by tectonic stress has never been revealed and quantitatively supported. Herein, according to the identified patterns by NMF, we can make a forward step to deeply understand the process about how tectonic stress affects ground deformation.

In natural conditions, the maximum principal stress $\sigma_{1}$ is perpendicular to the strike of ore body(Ma et al. 2015). The long axis of strain ellipse is NW-SE and the relationship of stress is $\sigma_{1}$ $>\sigma_{2}$ (Fig. 6b). In post-mining condition, we find that the $\mathrm{Z}$ displacement at the center of ground deformation in S2 pattern can be simplified as two sources controlled by S1 and S3 because the contribution of S2 is nearly zero. Therefore, the ellipse shape at the center region (Fig. 5b) can be thought as strain ellipse and the long axis is NE-SW. The rotation of ellipse indicates that mining operations has caused the stress rotation in a larger area. At the same time, the relationship of stress changes to $\sigma_{2}>\sigma_{1}$. This can be supported by deformation traces. In detail, by overlying S1 and S3 patterns, we find a set of "x" shape strong deformation belts on the surface (Fig. 6a). Through the deformation traces, it is evident that the deformation belt by S3 (left wing) shows a shear property, and the slide direction can be obtained to reveal $\sigma_{2}>\sigma_{1}$. The two quasi-circular closed region at each end of shear belt may be caused by stress concentration (Fig. 5c)(Guo et al. 2020). Deformation belt by $\mathrm{S} 1$ (right wing) shows an extension property because the displacement contour line in cross section of " $\mathrm{x}$ " is straight and parallel to each other. In fact, as the mining tunnel is perpendicular to 
fractures perpendicular to $\sigma_{1}$ on surface (field investigation can be seen in Ma et al. (2015)) and constraint of $Y$ displacement by increasing the normal stress on joint planes. As a result, $\sigma_{1}$ causes a large scale of $X$ displacement at the beginning, and, then, $\sigma_{2}$ becomes the dominate stress in the disturbed stress field which dominates $\mathrm{Y}$ displacement. This results in that S1 and S3 explain different volume information of original data (Table 2 ) with the explanation ratios of $\xi_{1} / \xi_{3}=1.05$. It is worth noting that the sum of explanation $\xi_{1}, \xi_{2}$, and $\xi_{3}$ is larger than 1 because these three sources are not orthogonal causing the existence of partial correlation. Therefore, we conclude that $\mathrm{S} 1$ is driven horizontal principal stress $\sigma_{2}$, and $\mathrm{S} 3$ is driven horizontal principal stress $\sigma_{1}$.

S2 describes a homogeneously and broadly distributed pattern that represents the self-weight body force caused by gravity. In the scale of study area, density of rock mass is the only factor affect the value and homogeneity of self-weight body force. We find that the mechanism of self-weight body force on ground deformation is significantly different from empirical knowledge. Specifically, on one hand, surface deformation induced by self-weight body force explains the least information of original data compared to S1 and S3. On the other hand, self-weight body force has a high contribution to $\mathrm{Z}$ displacement far away from center while low contribution near the subsidence center (Fig. 5b). Compression state of rock mass is found at the center of ground deformation in many studies (Ma et al. 2018; Xia et al. 2016). In this study, high confining pressure caused by $\sigma_{1}$ and $\sigma_{2}$ produces enough force of friction on joint planes with high dip angle that alleviating the $\mathrm{Z}$ displacement. At the same time, $\mathrm{Z}$ displacement is dominated by $\sigma_{1}$ and $\sigma_{2}$ because of the existence of the joint planes with lower dip angle. This can be supported by ratios of source contribution on single point such as 14 7 and $\mathrm{H} \sim 11$ (Fig. 9c). Besides, we propose that the high contribution of $\mathrm{S} 2$ away from the center may be caused by the extension-subsidence after the stress release of $\sigma_{1}$ and $\sigma_{2}$.

\subsection{Temporal pattern of source}

Temporal evolution of displacement is critical for disaster early warning. However, previous studies focus on the displacement evolution responding to the combined actions of ambient sources( $\mathrm{Li}$ et al. 2020b). In contrast, in this study, temporal evolution of each displacement component is related to separate source. Fig. 7 shows the temporal pattern of three sources. The temporal pattern does not show periodicity or seasonality related to precipitation indicating that the precipitation induced consolidation deformation can be ignored in this arid region. Another important conclusion from the analyses is that the time lag is not obvious by comparing the curve of three sources (Fig. 7). This indicates these three sources are independent in physical mechanism.

The deformation rate of three sources slowly increases with time. In detail, S1 induces the largest increment of deformation rate of 0.0094 , and S3 induces a medium value of 0.0086 , and S2 causes a lowest increment of deformation rate of 0.0056 (Fig. 7). The different increment of deformation rate may be driven by the different physical process. The stress concentration is more remarkable with mining depth increase, and, thus, the displacement controlled by $\sigma_{1}$ and $\sigma_{2}$ increases slowly. In contrast, the self-weight body force has no obvious change because it is controlled by gravity and the volume and density of overlying rock mass. Therefore, the increment of deformation rate caused by S2 would be a stable value ideally. However, the discontinuities, especially the joint and other structure planes, contribute to a component of $\mathrm{Z}$ displacement when suffering horizontal stress. As the dynamic of stress redistribution, the normal stress may decrease on structure planes at the tensile zone mainly distributed at the edge of deformation area (Fig. 5b) can favor the $\mathrm{Z}$ displacement driven by self-weight body force. 
By comparing the source contribution to $\mathrm{X}, \mathrm{Y}$, and $\mathrm{Z}$ displacement, we can divide the temporal pattern into three stages (Fig. 8). These three stages reflect the evaluation of ground deformation. A "vibration-acceleration" phenomenon is revealed. During the vibration period, the stress redistribution and adjustment of discontinuities occur in rock mass. The acceleration is a relatively faster process, which is driven by the stress concentration and the degradation of rock mass. Another phenomenon is that each source has a more distinct contribution to its controlling displacement component while keeps a continuous decrease contribution to another two components. A reasonable explanation is that the overlying rock mass is becoming loose, and the confining pressure is decreasing on the plane of discontinuities. Therefore, $\mathrm{S} 3$ contributes more $\mathrm{X}$ displacement and less $\mathrm{Y}$, and $\mathrm{Z}$ displacement along the plane ( $\mathrm{S} 1$ and $\mathrm{S} 2$ have the same mechanism with $\mathrm{S} 3$ ). This conclusion can be used to explain many collapse events in other mining areas (Feng et al. 2019; Waltham et al. 2011).

\subsection{Relationship between potential strong deformation belt and the change of "controlling} pattern"

Most of sudden disasters are evolved from a long-time slow and micro deformation and the location of final failure always controlled by the previously deformed belts. GPS has successfully applied to detect the slow slip and micro deformation of landslides and mining induced ground deformation (Gourmelen et al. 2007; Klein et al. 2018; LI Xiao 2020). Prediction of deformation is the main issue in previous studies(Chen et al. 2020). The main process is, firstly, fitting measured data by various methods and, then, predicting the deformation by learned models. However, few of these studies make a forward to reveal what drives the deformation. As we all know, ground deformation is always drove by multi sources in reality. Prediction may be more valuable and reliable on the basis of physical mechanism of each source. In this study, we assume that the GPS data is a mixture of a series of unknown sources with dynamic mixing ratios. As a result, we separate two horizontal tectonic stresses and self-weight body force controlling the characteristics and tendency of ground deformation.

On the basis of separated sources, we, firstly, propose a new conception "controlling pattern" that is defined as a combination of sources ordered by their contribution at a certain time. It is dynamic with time and any source can become the dominate source. It is known that different value of confining pressure can cause different shape and location of rupture in the triaxial compression test. Like that, different controlling patterns will lead to different features of ground deformation. In addition, the location of micro deformation by first controlling pattern will play an important role in subsequent deformation process like "buckets effect". Fig. 9 shows the contribution ratio of S1, $\mathrm{S} 2$, and $\mathrm{S} 3$ at each point. The relationship of three ratios is stable generally except for several points located on strong deformation belts. For X displacement, the dominate source is S3, and the secondary and the third is S1 and S2, respectively. In the strong deformation belts, the source contribution of S3 decreases sharply while the contribution of S1 increases at points, such as $8 \sim 3$, 10 9, 14-7, 18 6, H-11, and Q 1 (Fig. 9a, 9b). The trend of $\mathrm{Y}$ displacement is similar to $\mathrm{X}$ displacement. Transformation of dominate source is found in $\mathrm{Z}$ displacement (Fig. 9c). The dominate sources $\mathrm{S} 2$ transfers to $\mathrm{S} 1$ or $\mathrm{S} 3$ in strong deformation belts. In addition, these changes are the same trend in each monitoring campaigns, which means we can identify it at an early phase of ground deformation helping to prevent risk like the severe damage of ventilation shaft on line 14

\section{Discussion}




\subsection{Effect of discontinuity}

Displacement is the comprehensive result that can be treated as a transient signal on the surface without caring about the transmit process in rock mass. Here, it is important to emphasize that in the presented analysis, we do not estimate the source transients changed during their propagation, and the effect of many influence factors before the displacement reaching the monitoring point. The NMF only decomposes the manifestation of the source transients in the observed data. Many driving sources and influence factors affect the development of ground deformation(Peng et al. 2018). The driving sources are related to external force loadings that are the source of displacement. Influence factors include lithology and structure of rock mass, and some physical processes that affect the mechanical properties and geometry of rock mass and discontinuity. These influence factors are highly related to the characteristics of displacement components, which covers up the characteristics of driving sources. In general, it is difficult to characterize and separate the effect of each influence factor in a regional scale because we even not understand the mechanic of a single factor, much less the coupling mechanics of all factors. However, it is more meaningful for safety management to know the comprehensive effect of these influence factors. As a result, NMF successfully identify three driving sources and the corresponding spatial and temporal patterns that is a reflection of the comprehensive effect of these influence factors. It is worth noting that driving sources identification is a core step to analyze the mechanism and evolution of ground deformation because they are carriers of these influence factors.

From the analysis in section 4, we find that discontinuities such as joints and faults, may dominant effects on the deformation behaviors of the rock mass in study area. Although NMF cannot give the precise effect of discontinuities, a conclusion can be proposed that discontinuities significantly contribute to rock movement in study area. In fact, field investigations find that there are four groups of dominate joints (Fig. 10a, c) with an average of dip direction is $7.2^{\circ}$ (percent: $16.31 \%), 96.27^{\circ}(26 \%), 186.2^{\circ}(29.37 \%)$, and $289.5^{\circ}$ (15.61\%), respectively. This indicates that joints with $\mathrm{E}$ and $\mathrm{S}$ dip direction can significantly affect the ground deformation. The consistency between direction of principal stress (Fig. 6a) and $\mathrm{E}$ and $\mathrm{S}$ dip direction favors the shear movement of rock, therefore, causing the shear belt and extension belt on the ground (Fig. 6a). In addition, S1 and $\mathrm{S} 3$ have a high contribution of $Z$ displacement (Fig. 4) while the pattern of S2 shows that S2 has a small contribution to $\mathrm{X}$ and $\mathrm{Y}$ displacement (Fig. 4), which suggests that the discontinuities with high dip angle are highly developed and, therefore, the rock has a small slide component in $\mathrm{X}$ and $\mathrm{Y}$ direction. Field investigations also reveal the highly developed of joints with high dip angle. As shown in Fig. 10c, the average dip of four groups of joints is $59.6^{\circ}, 54.2^{\circ}, 64.2^{\circ}, 63^{\circ}$, respectively.

The temporal pattern of three sources are also affected by discontinuity. The characteristic of each stage in Fig. 8 is different. The amplitude of source continuously increases from the first stage to the third stage. For example, S3 has a normalized amplitude of 0.63 in the first stage and increases to 0.72 in the second stage, and 0.82 in the third stage (Fig. 11a). S1 and S2 have the similar changes in amplitude (Fig. 11b, c). In addition, the repeated mining operations can disturb tectonic stress causing the concentration or relaxation of stress but the self-weight body force remains stable. This leads to the oscillation amplitude of S2 is smaller than that of S1 and S3. These temporal patterns reveal that the overlaying rock mass is becoming increasingly sensitive to stress perturbations, which is consistent with the process closing to the volumetric expansion point in uniaxial compression test (Fig.12). During this process, the discontinuity becomes unstable and deforms easily under small stress perturbation, especially shear stress perturbation. However, NMF cannot 
predict when the ground deformation will evolve to failure (collapse of overlaying strata).

\section{Conclusions}

Our analyses demonstrate the applicability of a new inverse method for analysis of ground deformation based on NMF algorithm of BSS. The solution is optimized by newly formulated evaluation criteria to reduce the interference among sources and improve the resolution of each pattern. The unknown sources are identified from a set of GPS time series data without any information about the sources, underground mining conditions, and the physical processes impacting the displacement propagation through rock mass.

The NMF based method identifies three unique sources causing the observed data. These are listed in a descending order in terms of source contribution to original data: S1 (driven by horizontal principal stress $\sigma_{2}$ ), S3(driven by horizontal principal stress $\sigma_{1}$ ), and S2 (driven by self-weight body force caused by gravity). They appear to be proportionally manifested at the monitoring point. Relative contribution of each source remains the same order as $\mathrm{S} 1>\mathrm{S} 3>\mathrm{S} 2$ except for the monitoring points on strong deformation belts where the source contribution changes distinctly and even dominate source transformation occurs. Besides, each source has stable spatial pattern which is time-independent. This allows us to identify the potential risk area at an earlier stage. Finally, the NMF based method can be applied to any real problem where temporal system behavior is monitored at multiple locations.

Although NMF is useful to long-term and cyclical driving source, NMF cannot identify some instant driving sources like blasting vibrating load and seismic load. The data sampling frequency (interval: 0.5 year) affects the result of NMF and high frequency sampling may overcome this problem and show more details of sources.

\section{Acknowledgements}

We gratefully acknowledge our financial support from the National Science Foundation of China (Grant Nos. 41907174, 41831293, 41772324), and National Key Research Projects of China (2016YFC0402802). The data used in our analysis is included with and described in https://github.com/guhongyu18/grounddeformation.

\section{Credit author statement}

We outline all authors' individual contributions as follows:

Hongyu Gu: Conceptualization, Data curation, Formal analysis, Investigation, Visualization, Writing - original draft, Writing - review \& editing, and Funding.

Fengshan Ma: Funding, Investigation, Project administration, Resources, Supervision, Validation, and Writing - review \& editing.

Liangjun Lin: Validation, Formal analysis, Revise, and Visualization

Donghui Wang: Investigation, Validation.

Yongbo Tie: Investigation, Funding.

Weichang Chen: Writing - review \& editing

\section{Declaration of Competing Interest}

None.

\section{References}

Bertin N, Badeau R, Richard G Blind Signal Decompositions for Automatic Transcription of Polyphonic Music: NMF and K-SVD on the Benchmark. In: Acoustics, Speech and Signal Processing, 2007. ICASSP 2007. IEEE International Conference on, 2007. 
Bibo D, Yongbin J (2016) Research on Distribution of Geometric Parameters of Structural Plane Metal Mine 4: 58-61.

Boutsidis C, Gallopoulos E (2007) SVD based initialization: A head start for nonnegative matrix factorization Pattern Recognition 41:1350-1362

Cai MQ (1999) Results and analysis of insitu stress measuremet at deep position of No.2 miningarea of Jinchuan Nichkel Mine Chinese Journal of Rock Mechanics \& Engineering 5:414-418

Cao J, Ma F, Guo J, Lu R, Liu G (2019) Assessment of mining-related seabed subsidence using GIS spatial regression methods: a case study of the Sanshandao gold mine (Laizhou, Shandong Province, China) Environmental Earth Sciences 78:26 doi:10.1007/s12665-0188022-1

Chang C, Fung PCW, Hung YS On a Sparse Component Analysis Approach to Blind Source Separation. In, Berlin, Heidelberg, 2006. Independent Component Analysis and Blind Signal Separation. Springer Berlin Heidelberg, pp 765-772

Chen B, Gong H, Li X, Lei K, Lin Z, Gao M, Zhou C (2017) Characterization and causes of land subsidence in Beijing, China International Journal of Remote Sensing 38:808-826

Chen B, Li Z, Yu C, Fairbairn D, Kang J, Hu J, Liang L (2020) Three-dimensional time-varying large surface displacements in coal exploiting areas revealed through integration of SAR pixel offset measurements and mining subsidence model Remote Sensing of Environment 240:111663 doi:https://doi.org/10.1016/j.rse.2020.111663

Ciaramella A, Lauro ED, Martino SD, Falanga M, Tagliaferri R (2006) ICA based identification of dynamical systems generating synthetic and real world time series Soft Computing 10:587606

Cohen-Waeber J, Bürgmann R, Chaussard E, Giannico C, Ferretti A (2018) Spatiotemporal Patterns of Precipitation-Modulated Landslide Deformation From Independent Component Analysis of InSAR Time Series Geophysical Research Letters 45:1878-1887 doi:10.1002/2017gl075950

Cuss CW, Guéguen C, Andersson P, Porcelli D, Kutscher L (2016) Advanced Residuals Analysis for Determining the Number of PARAFAC Components in Dissolved Organic Matter Applied Spectroscopy 70:1-13

Díaz-Fernández ME, Álvarez-Fernández MI, Álvarez-Vigil AE (2010) Computation of influence functions for automatic mining subsidence prediction Computational Geosciences 14:83103 doi:10.1007/s10596-009-9134-1

Ding K, Ma F, Guo J, Zhao H, Lu R, Liu F (2017) Investigation of the Mechanism of Roof Caving in the Jinchuan Nickel Mine, China Rock Mechanics and Rock Engineering 51:1215-1226 doi:10.1007/s00603-017-1374-0

Fathi Salmi E, Nazem M, Karakus M (2017) Numerical analysis of a large landslide induced by coal mining subsidence Engineering Geology 217:141-152 doi:https://doi.org/10.1016/j.enggeo.2016.12.021

Feng X, Zhang N, Xue F, Xie Z (2019) Practices, experience, and lessons learned based on field observations of support failures in some Chinese coal mines International Journal of Rock Mechanics and Mining Sciences 123:104097 doi:https://doi.org/10.1016/j.jirmms.2019.104097

Gourmelen, Noel, Amelung, Falk, Riccardo (2007) Mining-related ground deformation in Crescent Valley, Nevada: Implications for sparse GPS networks Geophysical Research Letters 34:252-254

Guo S, Qi S, Zhan Z, Ma L, Gure EG, Zhang S (2020) Numerical study on the progressive failure of heterogeneous geomaterials under varied confining stresses Engineering Geology 269:105556 doi:https://doi.org/10.1016/j.enggeo.2020.105556

Hoyer PO (2004) Non-negative matrix factorization with sparseness constraints Journal of Machine Learning Research 5:1457-1469 
Hui X, Ma F, Zhao H, Xu J (2018) Monitoring and statistical analysis of mine subsidence at three metal mines in China Bulletin of Engineering Geology and the Environment:3983-4001

Kabwe E (2017) Mining Sequence Deformation and Failure Behaviour Analysis in the hangingwall and Orebody Rock Formations; A Continuum Approach Geotechnical and Geological Engineering 35:1453-1473 doi:10.1007/s10706-017-0187-y

Karoui MS, Deville Y, Hosseini S, Ouamri A A new spatial sparsity-based method for extracting endmember spectra from hyperspectral data with some pure pixels. In: Geoscience \& Remote Sensing Symposium, 2012 IEEE International IEEE, 2012.

Khanal M, Adhikary D, Jayasundara C, Rao B (2015) Numerical Study of Mine Site Specific Multiseam Mining and Its Impact on Surface Subsidence and Chain Pillar Stress Geotechnical \& Geological Engineering 34:1-19

Klein E, Duputel Z, Zigone D, Vigny C, Boy J-P, Doubre C, Meneses G (2018) Deep Transient Slow Slip Detected by Survey GPS in the Region of Atacama, Chile Geophysical Research Letters 45:12,263-212,273 doi:10.1029/2018gl080613

Langville AN, Meyer CD, Albright R, Cox J, Duling D (2014) Algorithms, Initializations, and Convergence for the Nonnegative Matrix Factorization Eprint Arxiv

Lee DD, Seung HS (1999) Learning the parts of objects by non-negative matrix factorization Nature 401:788-791

Lee TW, Lewicki MS, Girolami M, Sejnowski TJ (1999) "Bind source separation of more sources than mixtures using overcomplete representations," Signal Processing Letters IEEE 6:87-90

Li G, Ma F, Guo J, Zhao H, Liu G (2020a) Study on deformation failure mechanism and support technology of deep soft rock roadway Engineering Geology 264:105262 doi:https://doi.org/10.1016/j.enggeo.2019.105262

Li M, Zhang L, Ding C, Li W, Luo H, Liao M, Xu Q (2020b) Retrieval of historical surface displacements of the Baige landslide from time-series SAR observations for retrospective analysis of the collapse event Remote Sensing of Environment 240:111695 doi:https://doi.org/10.1016/j.rse.2020.111695

Li X, Wang SJ, Liu TY, Ma FS (2004) Engineering geology, ground surface movement and fissures induced by underground mining in the Jinchuan Nickel Mine Engineering geology 76:93107

LI Xiao ZN, SHENG Zhuping, LI Shouding, HAO Jianming (2020) Sliding mechanisms and fracture genesis of Jiweishan landslide in Wulong\#br\# chinese Journal of Rock Mechanics \& Engineering 39:1-12 doi:10.13722/j.cnki.jrme.2019.0453

Ma F, Gu H, Guo J, Lu R ( 2018) Analysis of ground deformation based on GPS in Sanshandao gold mine, China Journal of Nepal Geological Society 55:7-14

Ma F, Zhao H, Yuan R, Guo J (2015) Ground movement resulting from underground backfill mining in a nickel mine (Gansu Province, China) Natural Hazards 77:1475-1490

Minka TP (2001) Automatic choice of dimensionality forPCA Media Laboratory Perceptual Computing Section Technical Report 13:556-562

Peng X, Yu P, Zhang Y, Chen G (2018) Applying modified discontinuous deformation analysis to assess the dynamic response of sites containing discontinuities Engineering Geology 246:349-360 doi:https://doi.org/10.1016/j.enggeo.2018.10.011

Perrin J, Cartannaz C, Noury G, Vanoudheusden E (2015) A multicriteria approach to karst subsidence hazard mapping supported by weights-of-evidence analysis Engineering Geology 197:296-305 doi:https://doi.org/10.1016/j.enggeo.2015.09.001

Rezaei M, Boostani R (2013) Using the genetic algorithm to enhance nonnegative matrix factorization initialization Expert Systems 31:213-219

Rickard S, Cichocki A When is non-negative matrix decomposition unique? In: 42nd Annual Conference on Information Sciences and Systems, CISS 2008, Princeton, NJ, USA, 19-21 March 2008, 2008.

Sahu SP, Yadav M, Das AJ, Prakash A, Kumar A (2017) Multivariate statistical approach for 
assessment of subsidence in Jharia coalfields, India Arabian Journal of Geosciences 10:191 Salmi EF, Karakus M, Nazem M (2019) Assessing the effects of rock mass gradual deterioration on the long-term stability of abandoned mine workings and the mechanisms of postmining subsidence - A case study of Castle Fields mine Tunnelling and Underground Space Technology 88:169-185 doi:10.1016/j.tust.2019.03.007

Schultz-Fellenz ES et al. (2020) High-resolution surface topographic change analyses to characterize a series of underground explosions Remote Sensing of Environment 246:111871 doi:https://doi.org/10.1016/j.rse.2020.111871

Song S, Zhao X, Xie J, Guan Y (2011) Grey Correlation Analysis and Regression Estimation of Mining Subsidence in Yu-Shen-Fu Mining Area Procedia Environmental Sciences 10:17471752 doi:https://doi.org/10.1016/j.proenv.2011.09.274

Tangirala AK, Kanodia J, Shah SL (2007) Non-Negative Matrix Factorization for Detection and Diagnosis of Plantwide Oscillations Industrial \& Engineering Chemistry Research 46:801-817 Waltham T, Park HD, Suh J, Yu MH, Kwon HH, Bang KM (2011) Collapses of old mines in Korea Engineering Geology 118:29-36 doi:https://doi.org/10.1016/j.enggeo.2010.11.007

Xia K, Chen C, Fu H, Pan Y, Deng Y (2016) Mining-induced ground deformation in tectonic stress metal mines: A case study Bulletin of Engineering Geology \& the Environment 210:212-230

Xia K, Chen C, Zheng Y, Zhang H, Liu X, Deng Y, Yang K (2019) Engineering geology and ground collapse mechanism in the Chengchao Iron-ore Mine in China Engineering Geology 249:129-147 doi:https://doi.org/10.1016/j.enggeo.2018.12.028

Xue Y, Tong CS, Chen Y, Chen W-S (2008) Clustering-based initialization for non-negative matrix factorization Applied Mathematics \& Computation 205:525-536

Ye S, Yue L, Wu J, Yan X, Wang H, Xun J, Teatini P (2016) Three-dimensional numerical modeling of land subsidence in Shanghai, China Hydrogeology Journal 24:695-709

Zhao H, Ma F, Xu J, Guo J (2012) In situ stress field inversion and its application in mininginduced rock mass movement International Journal of Rock Mechanics and Mining Sciences 53:120-128 doi:10.1016/j.ijrmms.2012.05.005

Zhao H, Ma F, Zhang Y, Jie G (2013) Monitoring and mechanisms of ground deformation and ground fissures induced by cut-and-fill mining in the Jinchuan Mine 2, China Environmental Earth Sciences 68:1903-1911

Zhou C et al. (2019) Quantifying the contribution of multiple factors to land subsidence in the Beijing Plain, China with machine learning technology Geomorphology 335:48-61 doi:https://doi.org/10.1016/j.geomorph.2019.03.017 
Figures

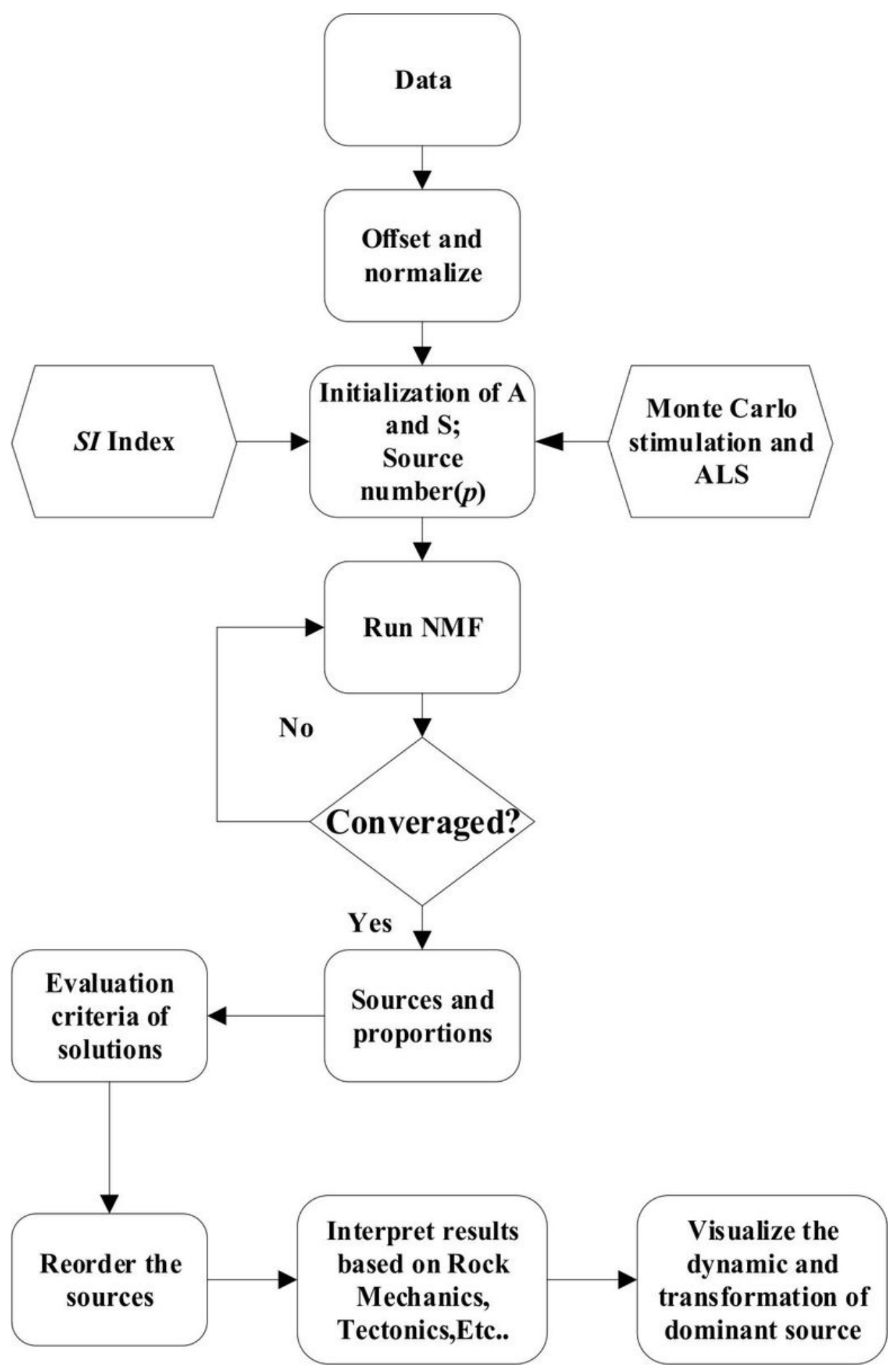

Figure 1

Flow chart of the BSS based on NMF algorithm. 


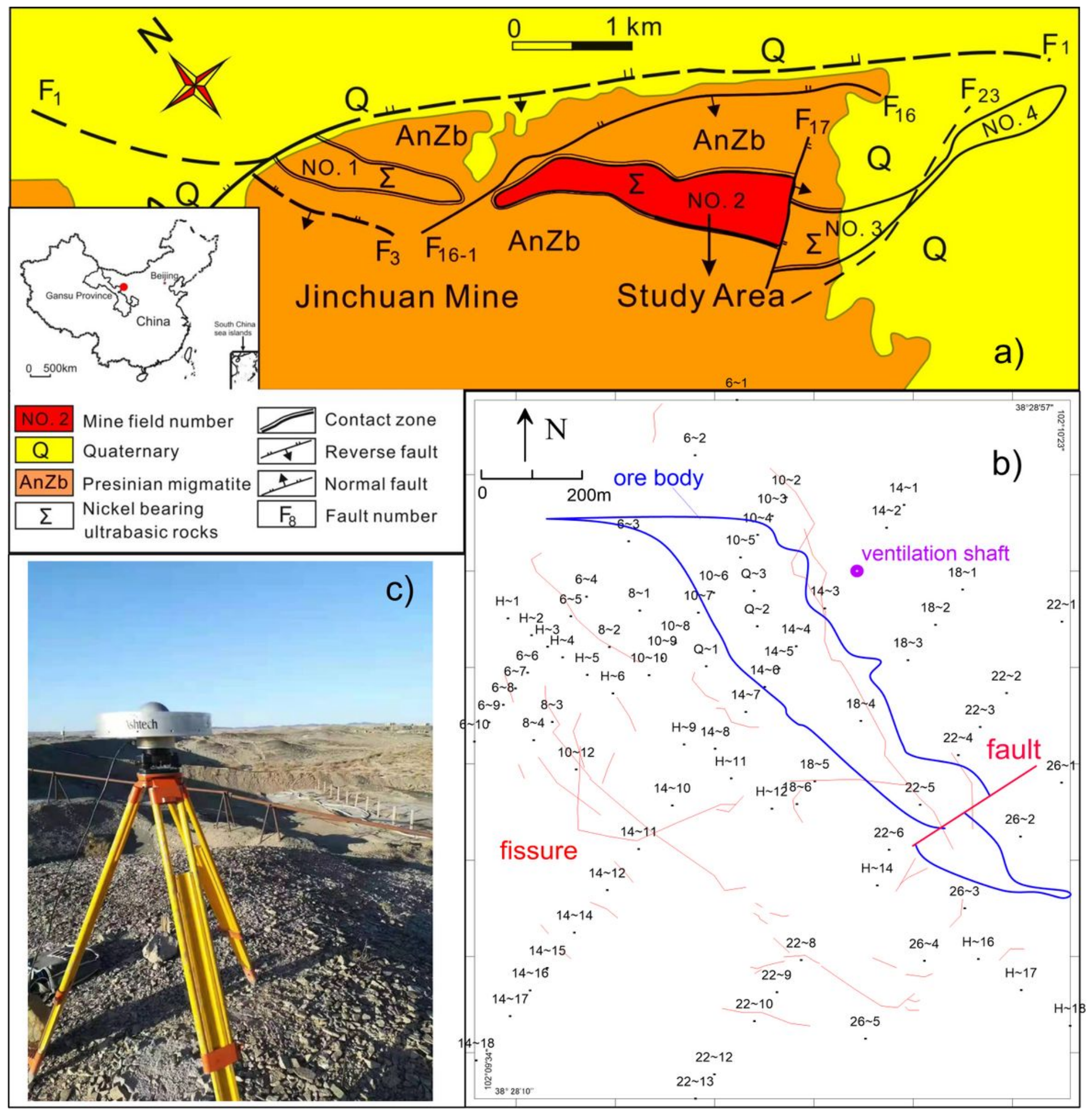

Figure 2

a) location of study area and geological setting, b) distribution of monitoring points, and c) the Z-12-type GPS receiver. Note: The designations employed and the presentation of the material on this map do not imply the expression of any opinion whatsoever on the part of Research Square concerning the legal status of any country, territory, city or area or of its authorities, or concerning the delimitation of its frontiers or boundaries. This map has been provided by the authors. 


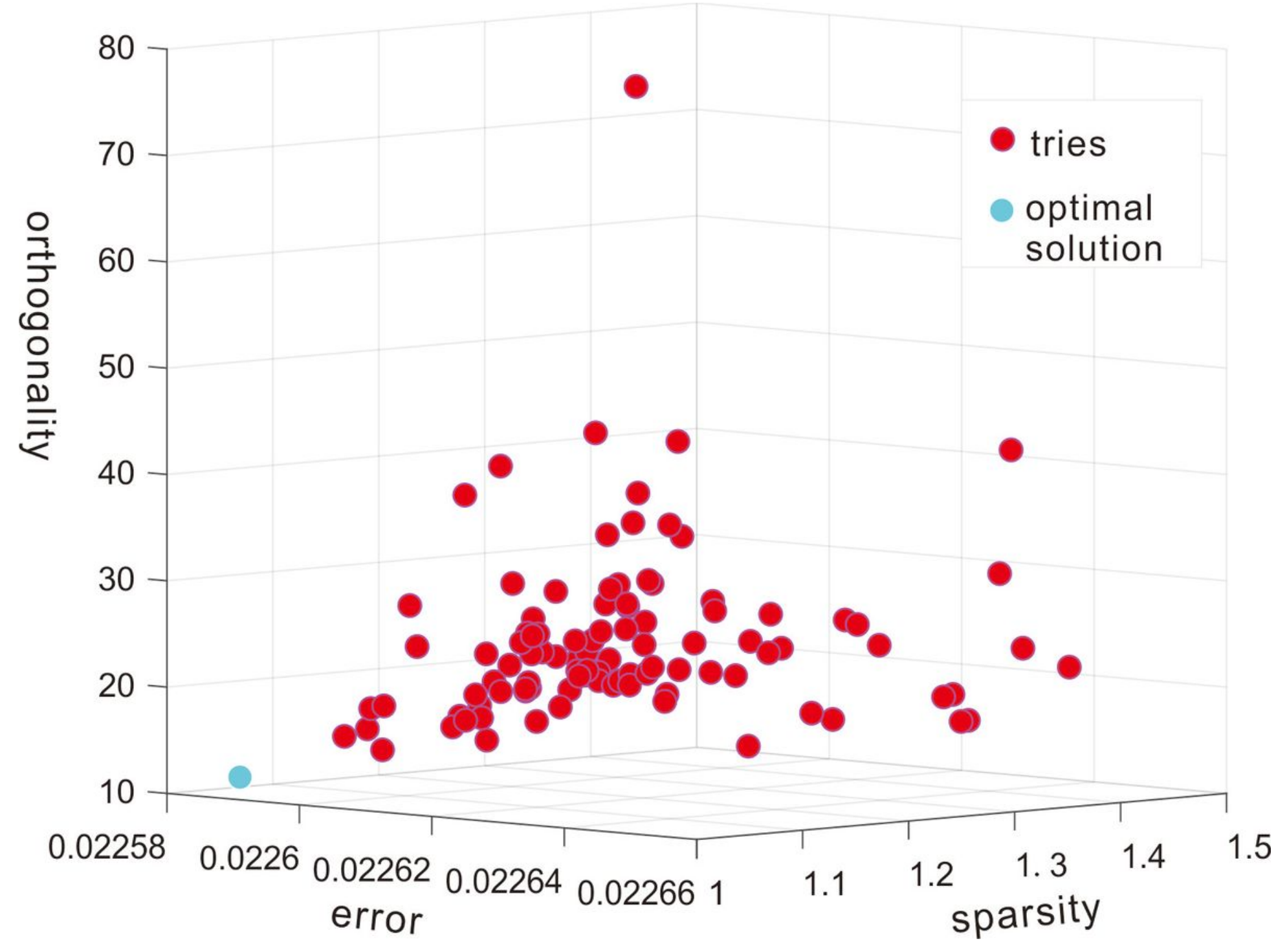

Figure 3

Results of 100 tries of NMF and the optimal solution is in blue. 


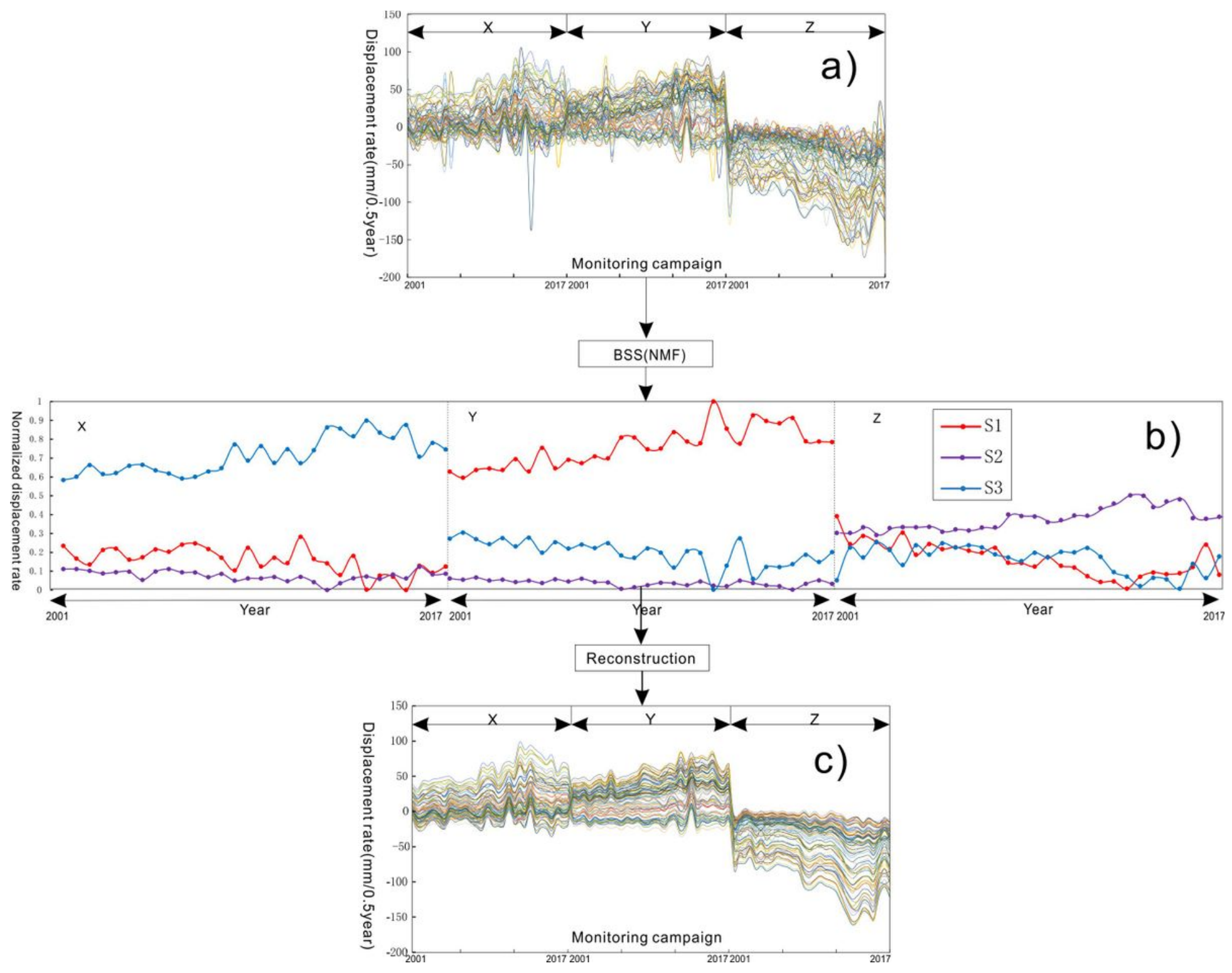

Figure 4

a) original data, b) the identified three sources by NMF, and c) the reconstructed data. 

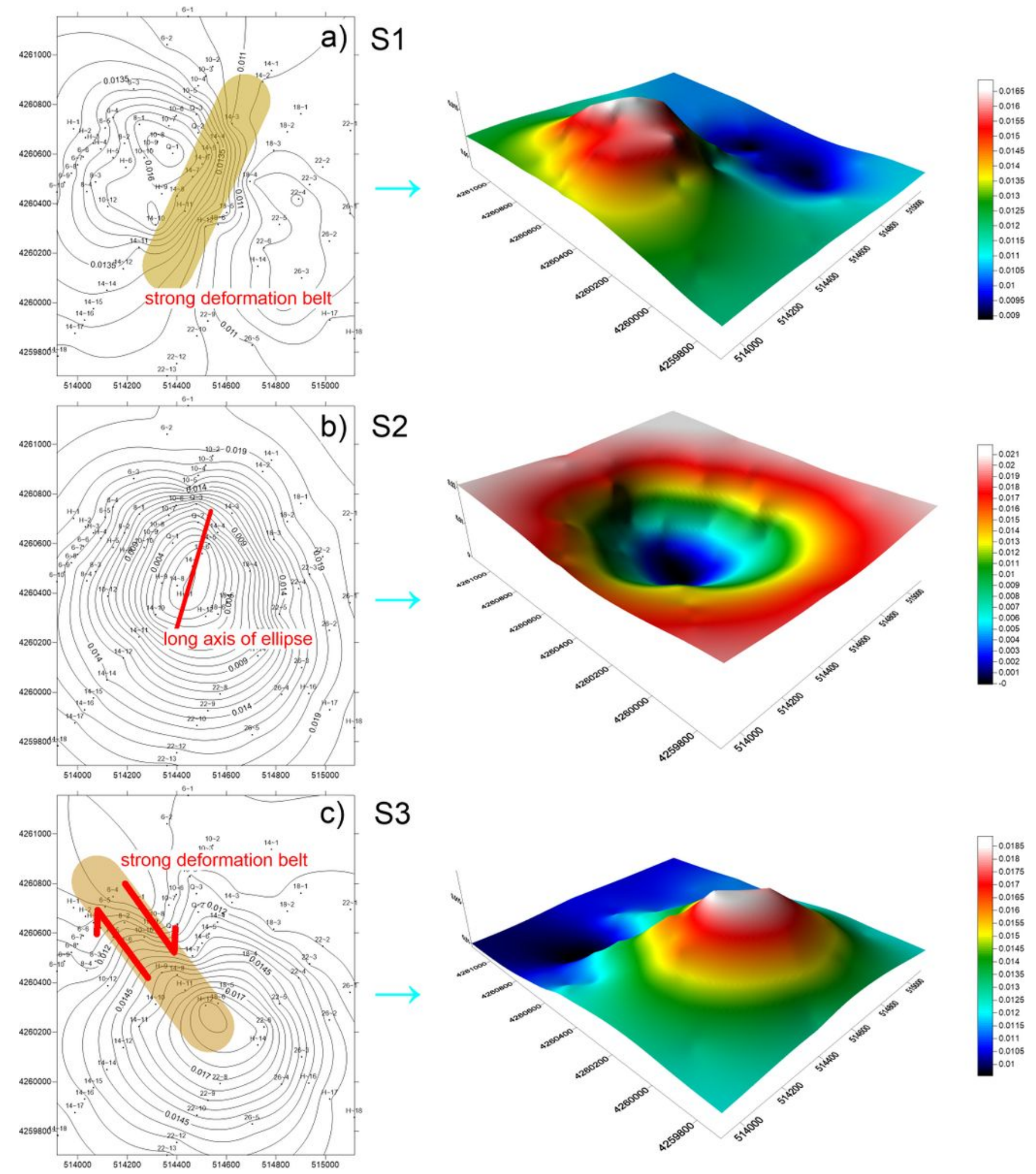

Figure 5

Spatial pattern of three sources including contour map and 3 dimensional graphics. Strong deformation belts are identified by deformation traces. 


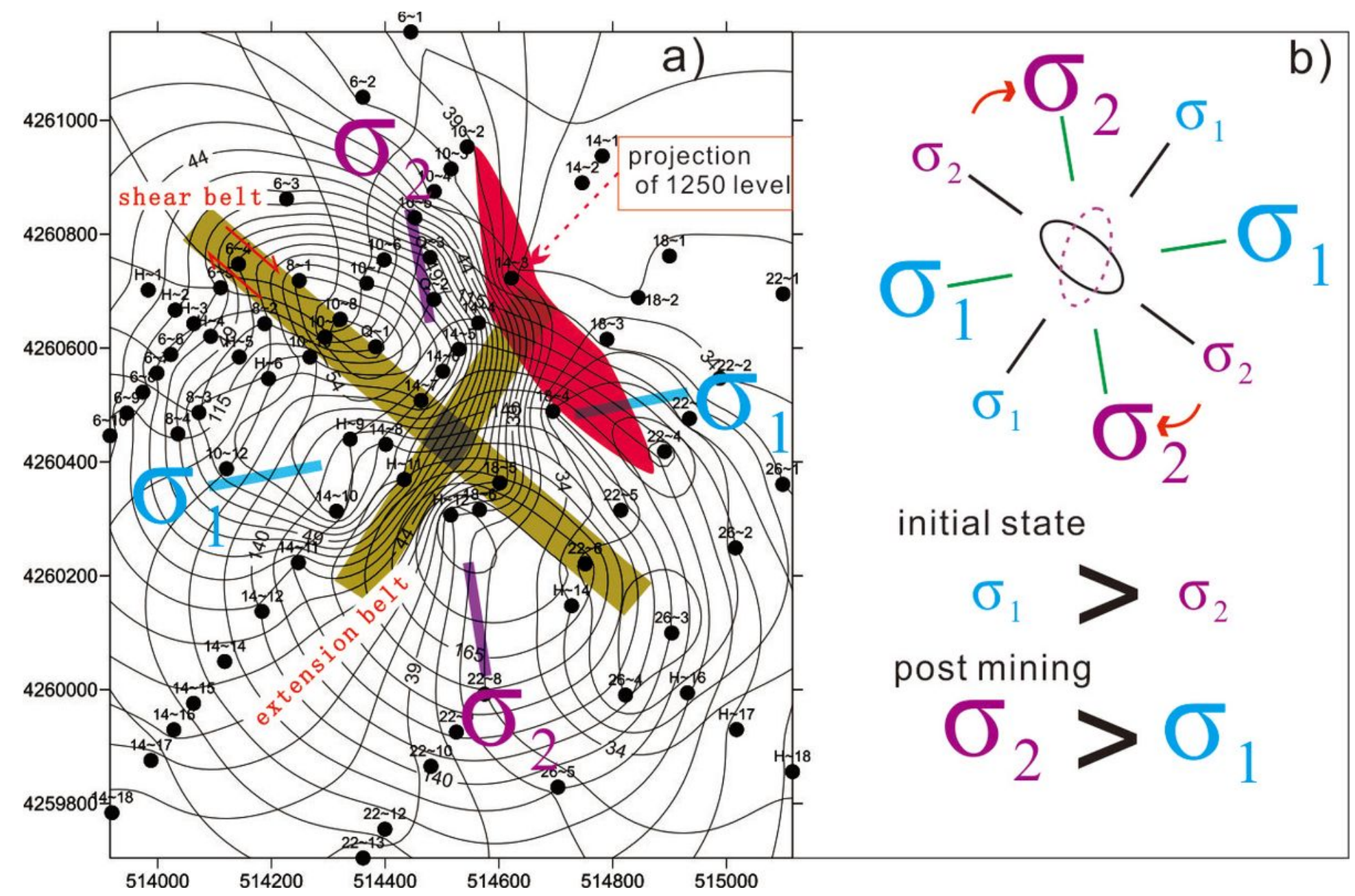

Figure 6

a) stress state is analyzed based on deformation traces by overlying the contour map of S1 and S3. b) stress state before mining and post mining, and the rotation of stress occurs after mining. 


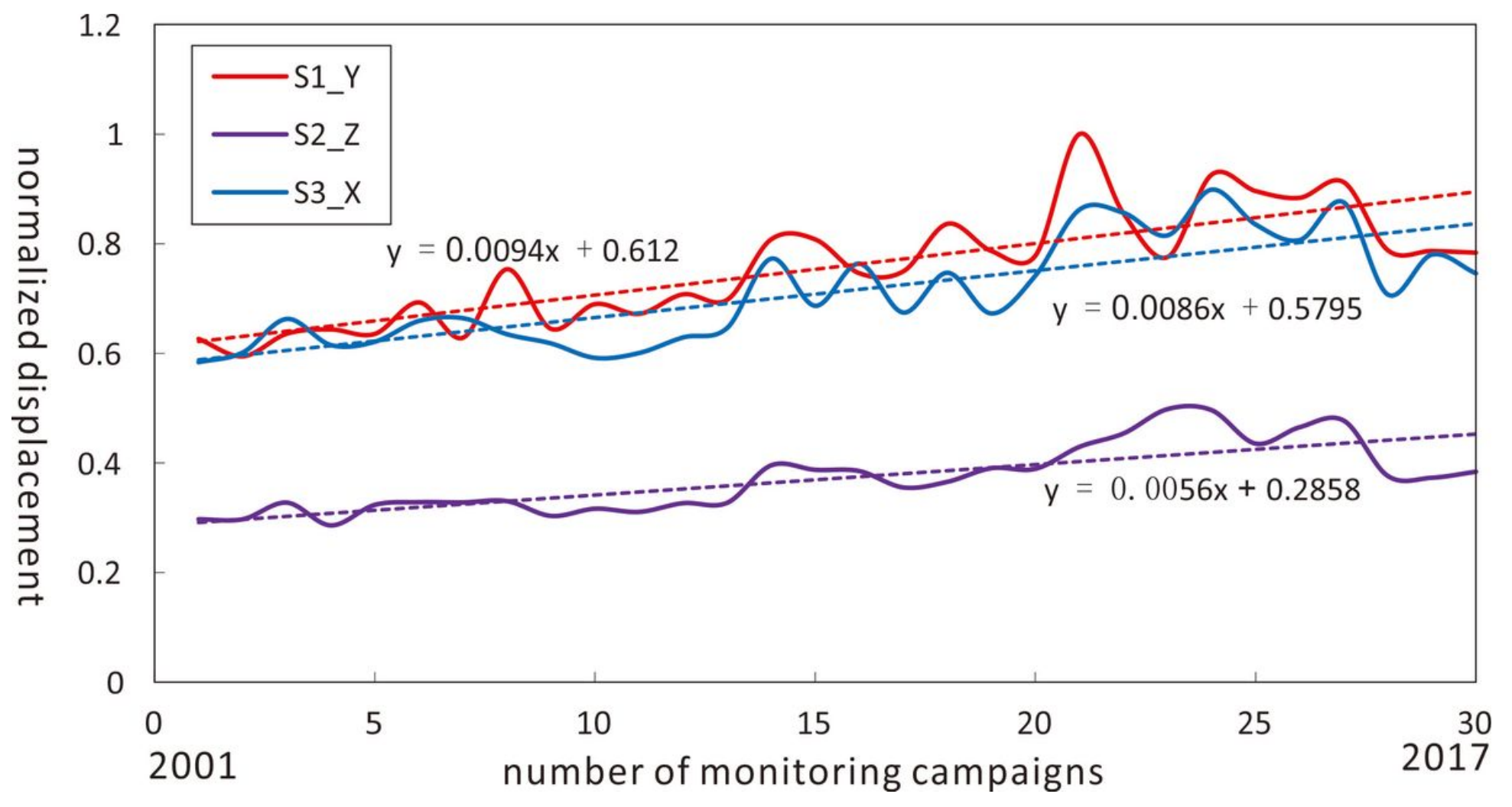

Figure 7

The temporal pattern of three sources. 


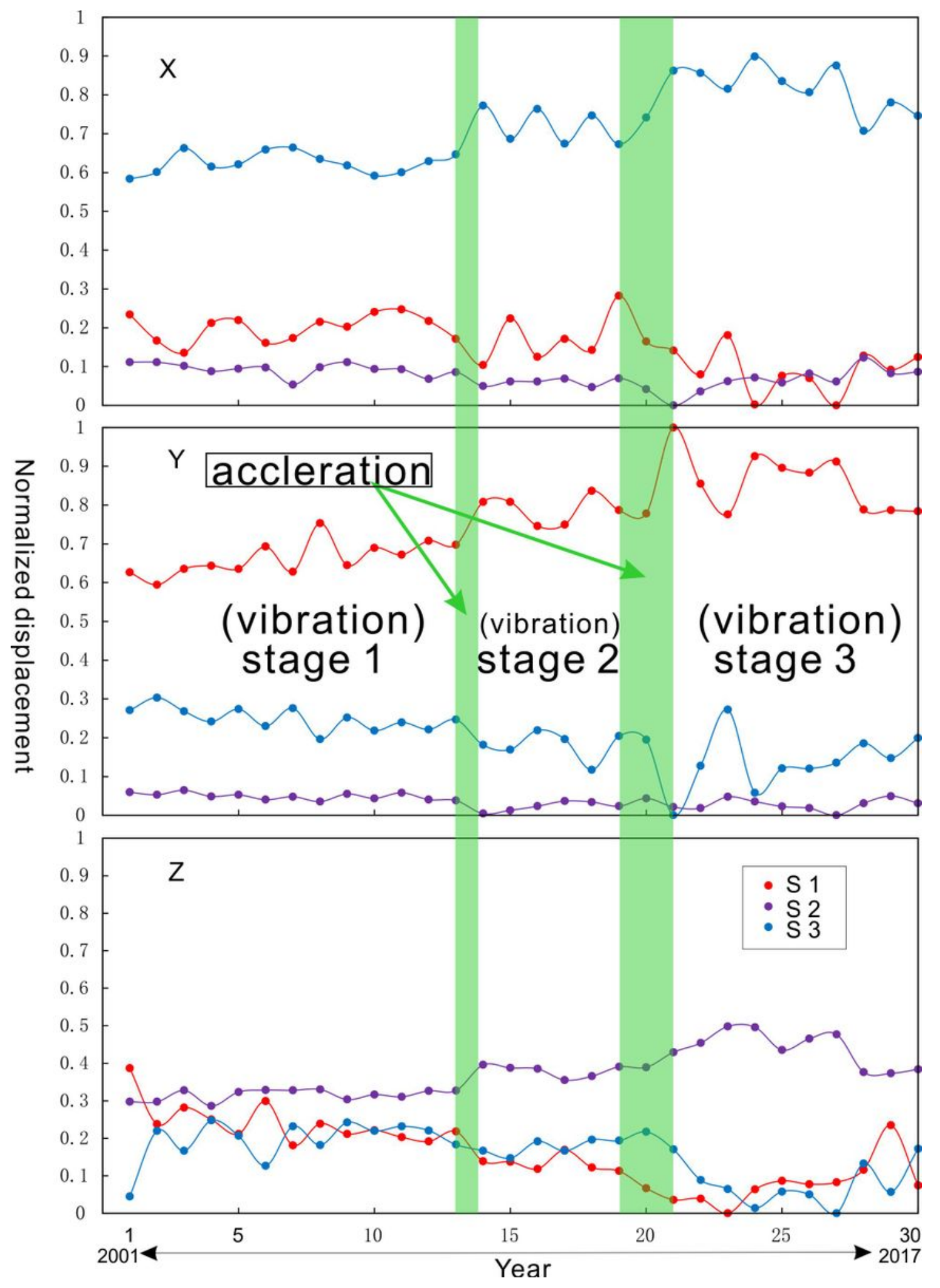

Figure 8

The evolution of ground deformation consisting of three stages of $X, Y$, and $Z$ displacement. $Y$ axis represents the normalized displacement. Dot represents the normalized displacement in a monitoring campaign. 

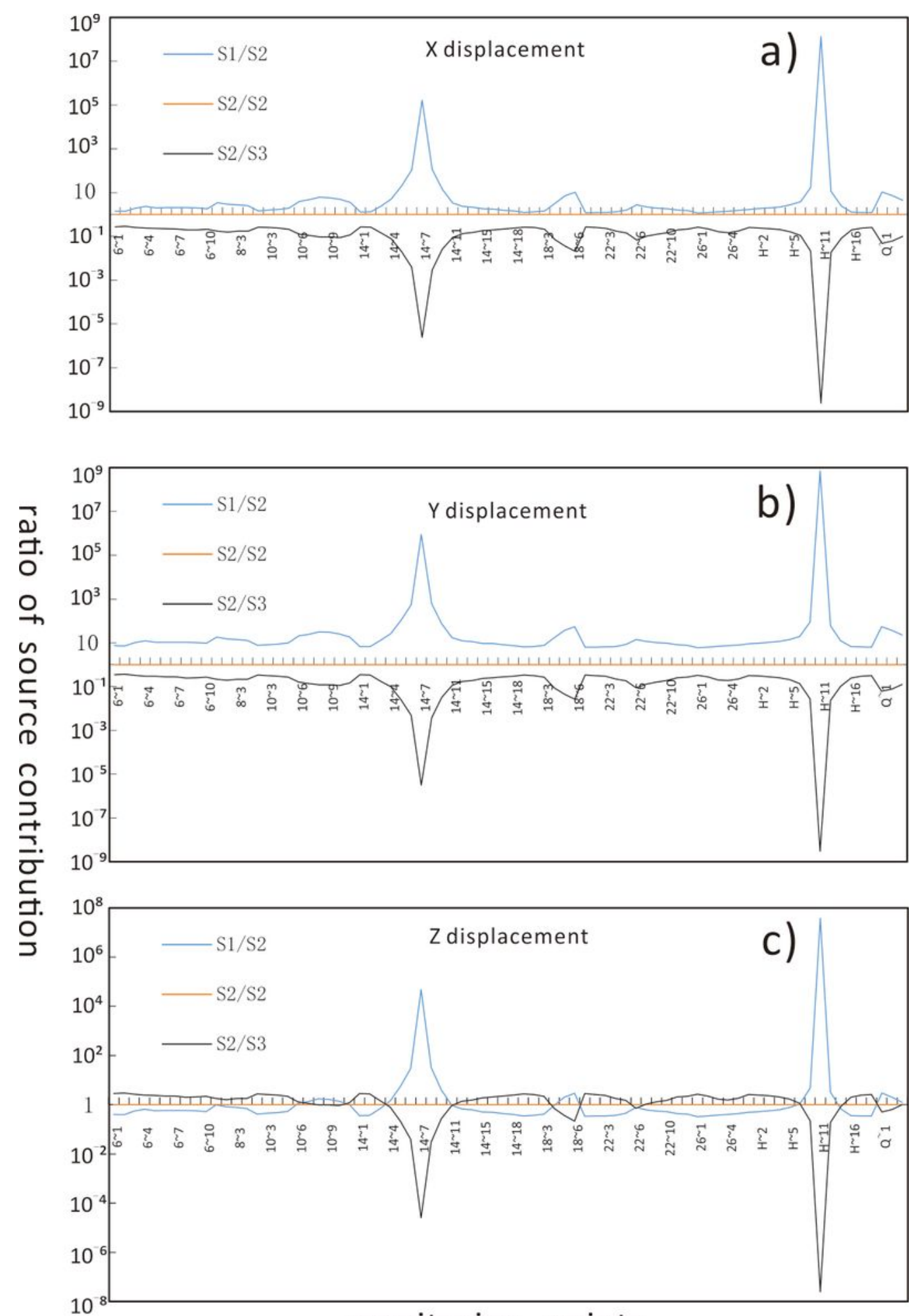

monitoring points

\section{Figure 9}

Dynamic of source contribution of $\mathrm{S} 1, \mathrm{~S} 2$, and $\mathrm{S} 3$ in $\mathrm{X}, \mathrm{Y}, \mathrm{Z}$ displacement, respectively. 

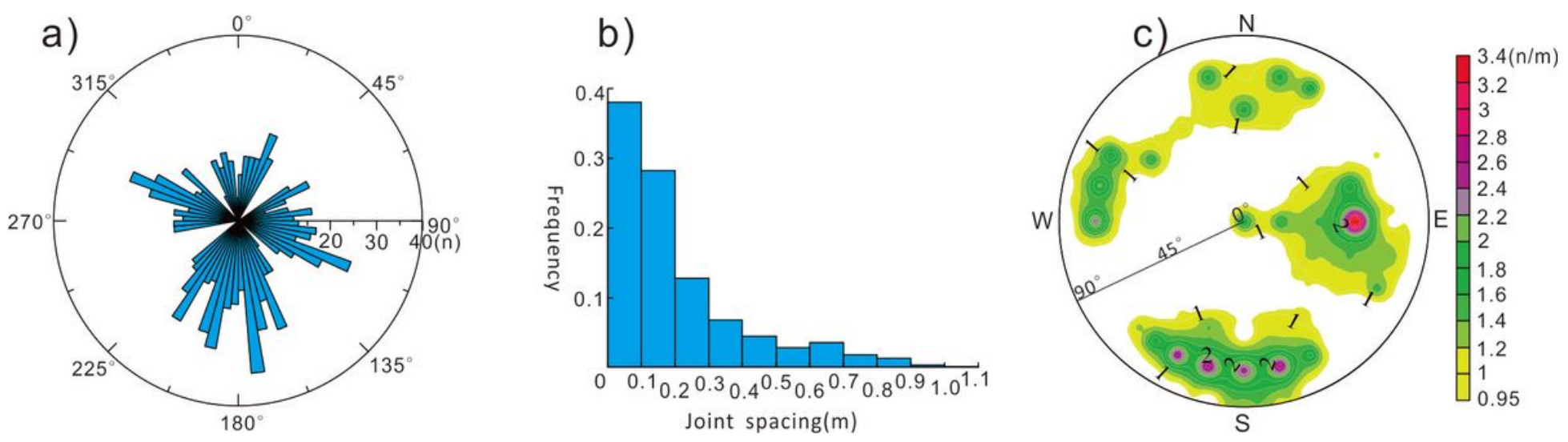

Figure 10

Statistical graphs of joints from $n=1134$ measured data (modified from Bibo et al. 2016). a) rose diagram of dip direction, b) histogram of joint spacing, c) joint density map.
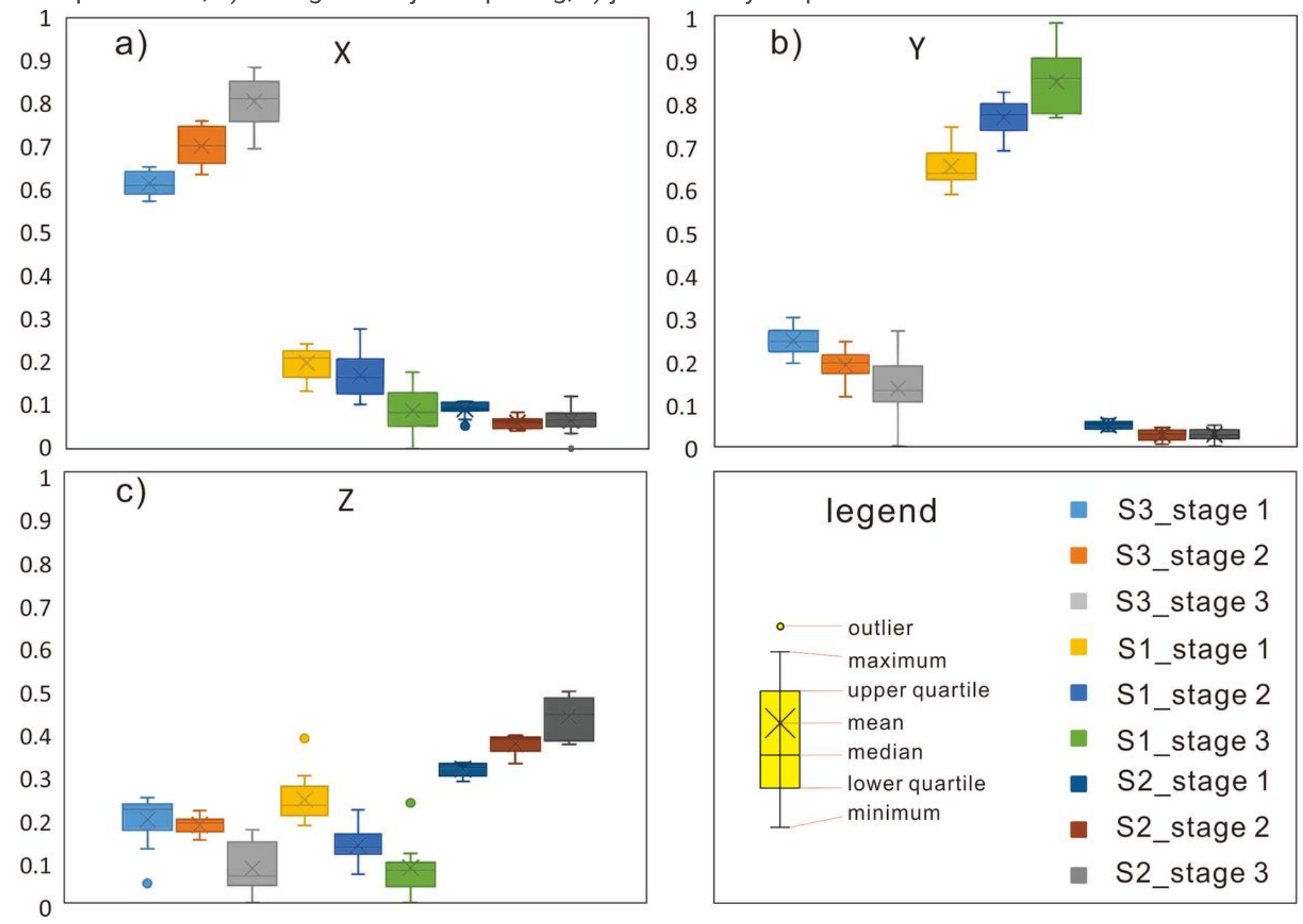

Figure 11

Boxplots of normalized oscillation amplitude of S1, S2, and S3 in three stages. 


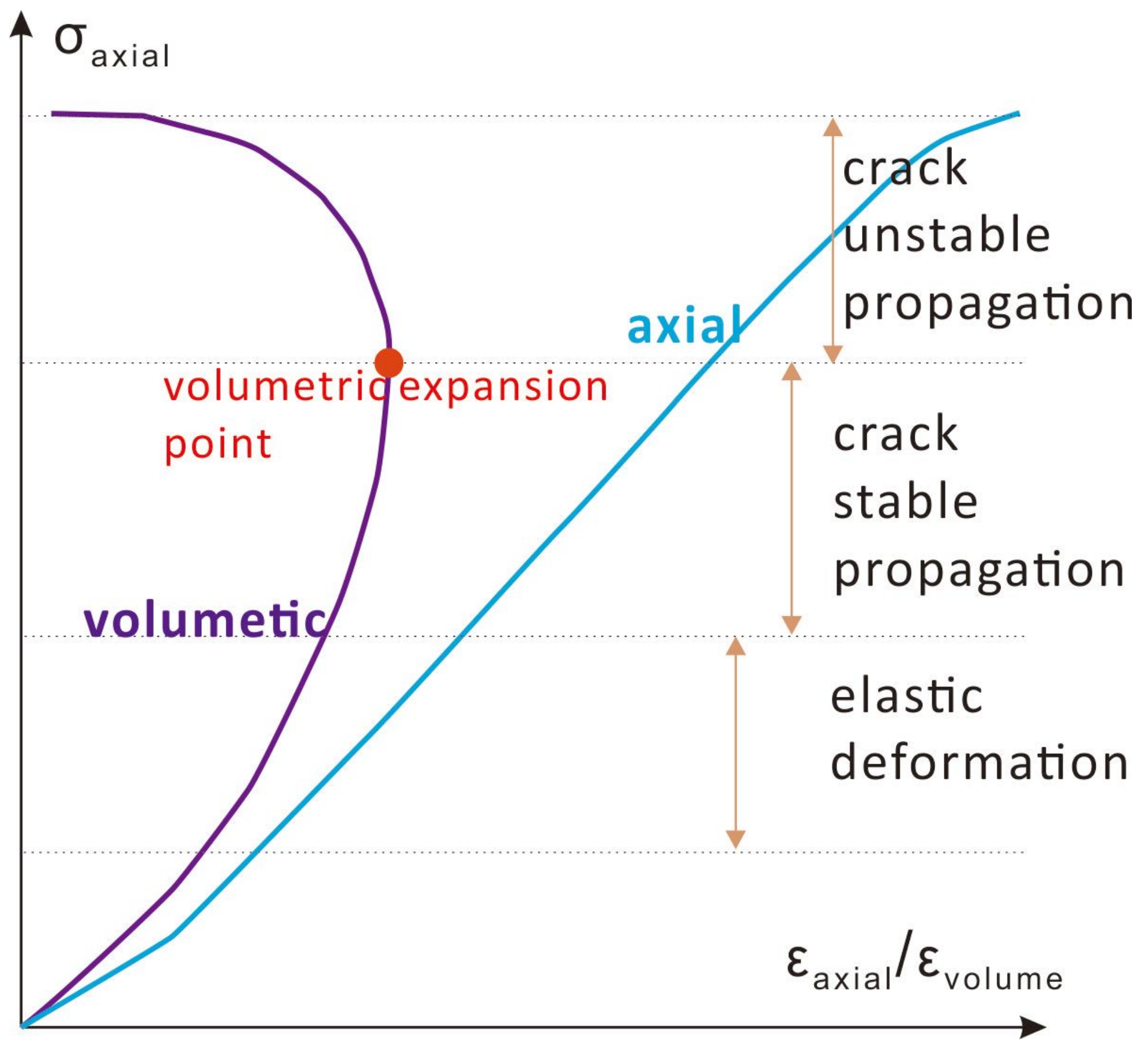

Figure 12

Schematic diagram of uniaxial compression test (axial stress vs ratio of axial strain to volume strain). 